\title{
Undamped transverse oscillations of coronal loops as a self-oscillatory process
}

\author{
V. M. Nakariakov ${ }^{1,2,3}$, S. A. Anfinogentov ${ }^{1}$, G. Nisticò ${ }^{1}$, and D.-H. Lee ${ }^{2}$ \\ ${ }^{1}$ Centre for Fusion, Space and Astrophysics, Department of Physics, University of Warwick, CV4 7AL, UK \\ e-mail: V.Nakariakov@warwick.ac.uk \\ 2 School of Space Research, Kyung Hee University, Yongin, 446-701 Gyeonggi, Korea \\ 3 Central Astronomical Observatory at Pulkovo of the Russian Academy of Sciences, 196140 St Petersburg, Russia
}

Received 4 May 2016 / Accepted 18 May 2016

\begin{abstract}
Context. Standing transverse oscillations of coronal loops are observed to operate in two regimes: rapidly decaying, large amplitude oscillations and undamped small amplitude oscillations. In the latter regime the damping should be compensated by energy supply, which allows the loop to perform almost monochromatic oscillations with almost constant amplitude and phase. Different loops oscillate with different periods. The oscillation amplitude does not show dependence on the loop length or the oscillation period.

Aims. We aim to develop a low-dimensional model explaining the undamped kink oscillations as a self-oscillatory process caused by the effect of negative friction. The source of energy is an external quasi-steady flow, for example, supergranulation motions near the loop footpoints or external flows in the corona.

Methods. We demonstrate that the interaction of a quasi-steady flow with a loop can be described by a Rayleigh oscillator equation that is a non-linear ordinary differential equation, with the damping and resonant terms determined empirically.

Results. Small-amplitude self-oscillatory solutions to the Rayleigh oscillator equation are harmonic signals of constant amplitude, which is consistent with the observed properties of undamped kink oscillations. The period of self-oscillations is determined by the frequency of the kink mode. The damping by dissipation and mode conversion is compensated by the continuous energy deposition at the frequency of the natural oscillation.

Conclusions. We propose that undamped kink oscillations of coronal loops may be caused by the interaction of the loops with quasi-steady flows, and hence are self-oscillations, which is analogous to producing a tune by moving a bow across a violin string.
\end{abstract}

Key words. Sun: oscillations - Sun: corona

\section{Introduction}

Kink oscillations of solar coronal loops have been intensively studied for almost two decades since their observational detection in the extreme ultraviolet (EUV) band by Aschwanden et al. (1999) and Nakariakov et al. (1999). It is commonly accepted that these oscillations are standing kink modes of coronal loops, with the periods determined by the loop length, its magnetic field, and the density of the plasma inside and outside the loop (e.g. Nakariakov \& Ofman 2001). This interpretation was based on the theory of fast magnetoacoustic modes of coronal loops modelled as field-aligned plasma cylinders that was developed by Zaitsev \& Stepanov (1982) and Edwin \& Roberts (1983). Alternative models interpret kink oscillations as the magnification of photospheric motions by the sensitivity of the equilibrium magnetic topology to a small footpoint perturbation (Schrijver \& Brown 2000), displacements of coronal loops by fast wave trains created by geometrical dispersion in the stratified atmosphere (Uralov 2003), or a pattern of interference fringes produced by fast magnetoacoustic waves guided by a coronal arcade (Hindman \& Jain 2014). A recent statistical study, however, demonstrated that the oscillation period scales with the length of the oscillating loop (Goddard et al. 2016). This finding supports the interpretation, at least in the majority of observed cases, in terms of a model invoking fast magnetoacoustic eigen modes of individual coronal loops or their bundles.
Kink oscillations of loops are detected to appear in two different regimes: large-amplitude rapidly decaying oscillations and small-amplitude undamped oscillations (Wang et al. 2012; Nisticò et al. 2013). In the former regime the loop displacement reaches several minor radii of the loop and, typically, the oscillation decays in a few oscillation cycles. In the latter regime the amplitude is usually smaller than the minor radius of the loop, and remains constant or low-varying for a number of oscillation cycles (Anfinogentov et al. 2013, 2015). The same loop may oscillate in both these regimes, that is before and after some impulsive excitation (Nisticò et al. 2013). Interestingly, the oscillation period remains the same in both regimes.

Damping of kink oscillations is commonly attributed to coupling of the collective kink oscillation with Alfvénic torsional motions localised in the vicinity of a narrow resonant layer (e.g. Ruderman \& Roberts 2002; Goossens et al. 2002). Recent results demonstrated that the oscillation amplitude experiences either exponential or Gaussian time decay (Hood et al. 2013; Pascoe et al. 2016), however in a number of cases it is difficult to distinguish between these two scenarios.

Large-amplitude, rapidly decaying kink oscillations of coronal loops are usually excited by low coronal eruptive events that mechanically displace them from equilibrium (Zimovets \& Nakariakov 2015). Small-amplitude undamped kink oscillations are observed continuously without any visible driver. Similar to the oscillations in the decaying regime, the 


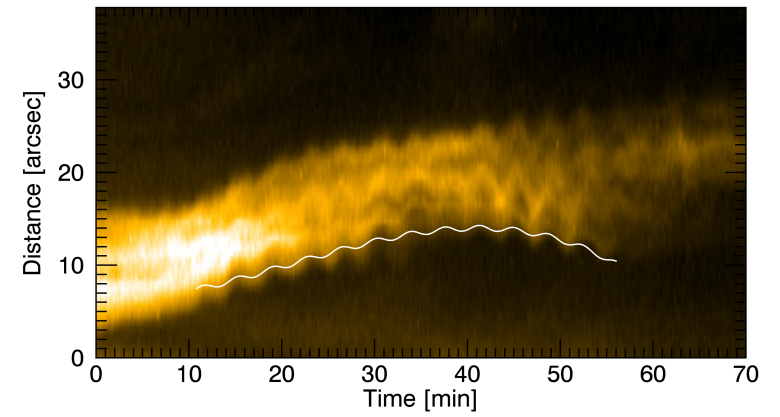

Fig. 1. Example of undamped oscillations of coronal loops, illustrated by a time distance map made for a slit directed across the oscillating loop. The oscillation was measured on 8 March 2011, beginning at 19:40 UT in AR 11165 at $171 \AA$ with SDO/AIA.

period of undamped kink oscillations was found to scale with the loop length (Anfinogentov et al. 2015). Assuming that the small-amplitude undamped kink oscillations are subject to the same damping mechanism as the large-amplitude, rapidly decaying kink oscillations, there should be some mechanism that continuously supplies the oscillating loops with energy to compensate the damping.

In this Letter we discuss possible mechanisms that could lead to sustained undamped kink oscillations of coronal loops. We demonstrate that the observational properties of these oscillations are, in particular, consistent with the outcomes of a model accounting for the negative friction between the loop footpoints and surrounding super-granulation flows.

\section{Observational results}

Undamped oscillations are detected as periodic, almost harmonic displacements of coronal loops. In Fig. 1 we show an example of such a process that is detected at $171 \AA$ with the Atmospheric Imaging Assembly on the Solar Dynamics Observatory (SDO/AIA). This oscillation was first analysed by Wang et al. (2012). During the oscillation, its amplitude experiences some variation, however it is typically much weaker and at much longer timescale than in the rapidly decaying regime. Sometimes up to 10-20 cycles of oscillations with almost constant amplitude are observed (e.g. Wang et al. 2012; Nisticò et al. 2013). Using the observations of undamped oscillations in 21 non-flaring active regions (NOAA 11637-11657) observed in January 2013 in the $171 \AA$ channel of SDO/AIA (Anfinogentov et al. 2015), we constructed scalings of the oscillation amplitudes with the period and loop length (Fig. 2). It is evident that the displacement amplitude gradually increases with the length of the oscillating loop and oscillation period. In contrast, the velocity amplitude that is estimated as the displacement amplitude multiplied by $2 \pi$ and divided by the oscillation period, does not show any dependence on the length and period.

\section{Models based on driven oscillations}

Consider different models that could be responsible for the undamped oscillations of a damped oscillator. Nakariakov et al. (2009), Nisticò et al. (2013), and Anfinogentov et al. (2015) suggested describing the displacement $a(t)$ of the loop top by a driven damped harmonic oscillator equation,

$\frac{\mathrm{d}^{2} a(t)}{\mathrm{d} t^{2}}+\delta \frac{\mathrm{d} a(t)}{\mathrm{d} t}+\Omega_{\mathrm{K}}^{2} a(t)=f(t)$,
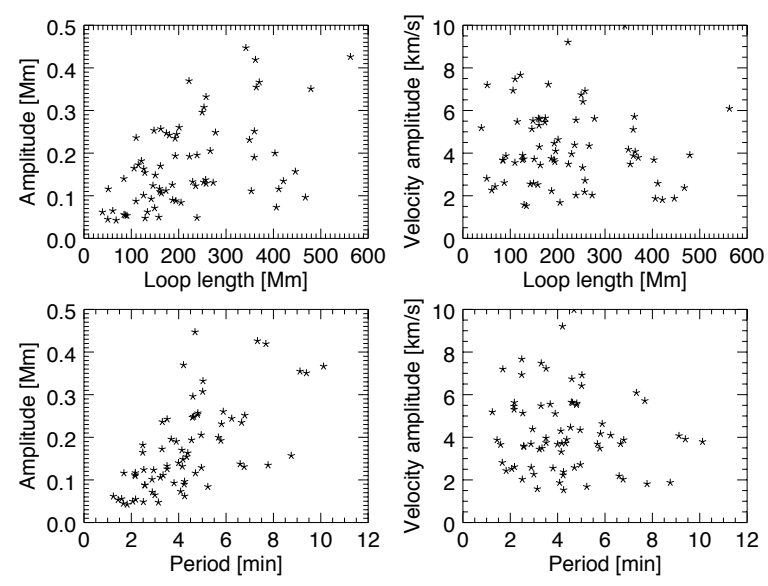

Fig. 2. Scalings of different parameters of undamped kink oscillations and oscillating loop parameters. Top left: displacement amplitude vs. loop length. Top right: velocity amplitude vs. loop length. Bottom left: displacement amplitude vs. oscillation period. Bottom right: velocity amplitude vs. oscillation period.

where $\delta$ is the damping coefficient, $\Omega_{\mathrm{K}}$ is the natural frequency of the kink oscillation, and $f(t)$ is an external driving force that compensates damping caused by dissipation and/or mode conversion. The damping coefficient can be obtained empirically from the large-amplitude, rapidly damped oscillatory regime. The excitation of loop oscillations by harmonic and random footpoint motions has also been addressed in 2D and 3D models (e.g. Ofman \& Davila 1995; Poedts \& Boynton 1996; Berghmans \& Tirry 1997). The most attention has been paid, however, to mode coupling and energy issues.

There are difficulties in reproducing the observed properties of undamped oscillations in terms of model (1) with a harmonic driver (i.e. 5 min oscillations of the loop footpoints). If the external force $f(t)$ is harmonic with the cyclic frequency $\omega_{0}$, as proposed in, for example Nisticò et al. (2013), the amplitude of the driven solution should depend on the difference between the driving force frequency and the natural frequency $\Omega_{\mathrm{K}}$. In the steady state, the oscillation occurs at the driving frequency and the driven oscillation amplitude is inversely proportional to the difference $\Omega_{\mathrm{K}}^{2}-\omega_{0}^{2}$. In other words, the amplitude of a harmonically driven, damped oscillator is a Lorentz curve, which has a maximum at the natural frequency $\Omega_{\mathrm{K}}$, but falls off rapidly for mismatching frequencies. Thus the loops with kink frequencies close to the driving frequency should show oscillations with the largest amplitudes. In other words, the scaling of the oscillation amplitudes with periods should have a pronounced peak. However, the observed dependence of the displacement and velocity amplitudes on the oscillation periods (Fig. 2) does not have any peak at a certain frequency. The frequency $\Omega_{\mathrm{K}}$ is prescribed, in particular, by the loop length (Edwin \& Roberts 1983), however, the scaling of the amplitude with the loop length does not have any peak either. Also, asymptotically, the damped driven oscillator oscillates with the frequency of the driver. It is not clear how the harmonic driver could have different, but stable frequencies ranging from $60 \mathrm{~s}$ to $600 \mathrm{~s}$, as is detected in observations (Anfinogentov et al. 2015).

Another possibility is the driving force to be a continuously operating random function, $f(t)=R(t)$, as it was suggest in, for example Anfinogentov et al. (2015) for the interpretation of decayless kink oscillations. The random driver could be associated with granulation motion near footpoints of the oscillating loop. This mechanism was considered in De Groof et al. (1998), 

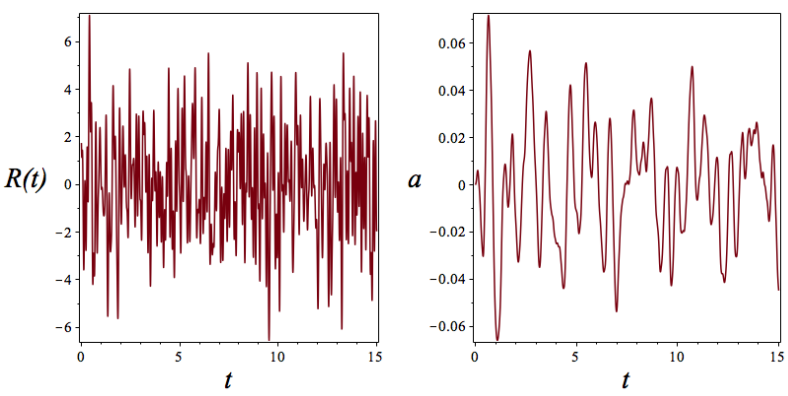

Fig. 3. Typical oscillations of a damped oscillator with a continuously operating random driver. Left: the random driving function. Right: the driven oscillation. The time is shown in units of oscillation periods, $2 \pi / C_{\mathrm{K}}$. The units of the vertical axes are arbitrary.

De Groof \& Goossens (2000) in the context of coronal heating. Figure 3 shows a typical response of a damped harmonic oscillator to random driving. It is evident that the behaviour of the loop is different from shown in Fig. 1; both oscillation phase and amplitude experience intermittent variation. A similar result was obtained by De Groof \& Goossens (2002) for a model that accounted for mode coupling. Interestingly, this behaviour resembles the regime studied in Nisticò et al. (2014), thus it may occur in the corona, but not in the regime discussed here.

In model (1), oscillations with the natural frequency could occur if the system is from time to time excited by short pulses. In this case, however, the resultant signal would be a series of exponentially decaying oscillation trains, which is not consistent with the observations either.

\section{Self-oscillatory model}

A possible way to compensate for the dissipation losses, while keeping the oscillation frequency and quasi-monochromaticity, is if the interaction of the loop with the surrounding moving medium is not "stiff", but slippery. Consider a mechanical analogue: a spring pendulum with the weight on a rough conveyor belt moving at a constant speed $v_{0}$ (Fig. 4). Its equation of motion is

$\frac{\mathrm{d}^{2} a(t)}{\mathrm{d} t^{2}}+\delta \frac{\mathrm{d} a(t)}{\mathrm{d} t}+\Omega_{\mathrm{K}}^{2} a(t)=F\left(v_{0}-\frac{\mathrm{d} a(t)}{\mathrm{d} t}\right)$,

where on the left-hand side we use the same notations as in Eq. (1) with the dissipative parameter $\delta$ accounting for, for example the dissipation in the spring. The function $F$ describes the friction between the load and the belt, which depends on their relative speed. Because of the dependence of $F$ on d $a / \mathrm{d} t$, Eq. (2) is, in general, non-linear. Making the Taylor expansion of the function $F$, we obtain the Rayleigh oscillator equation,

$\frac{\mathrm{d}^{2} a(t)}{\mathrm{d} t^{2}}-\left[\Delta-\alpha\left(\frac{\mathrm{d} a(t)}{\mathrm{d} t}\right)^{2}\right] \frac{\mathrm{d} a(t)}{\mathrm{d} t}+\Omega_{\mathrm{K}}^{2} a(t)=0$,

where $\Delta$ is the difference of the linear frictions with the belt and in the spring, and $\alpha$ a constant that depends on $v_{0}$ (e.g. Rayleigh 1945). In the Taylor expansion the linear term was set to zero, as it would result in a uni-directional friction force bias. By a simple substitution, Eq. (3) may be rewritten as the Van der Pol equation. Originally, Eq. (3) was derived by Lord Rayleigh for modelling the oscillations of a clarinet reed.

If the linear friction between the load and the belt is stronger than other dissipative processes, for instance in the spring, the

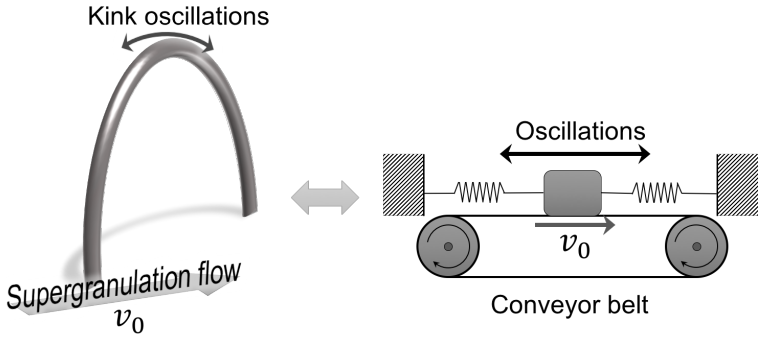

Fig. 4. Sketch of the proposed analogy between a coronal loop interacting with a supergranulation flow, a "string" and a "bow", respectively, and a spring pendulum on a steadily moving conveyor belt.
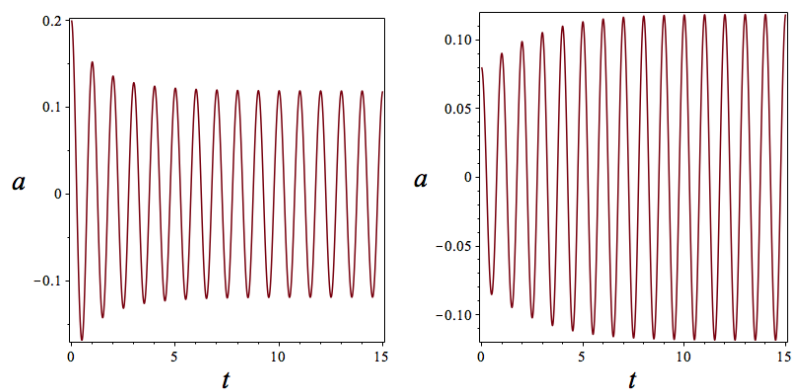

Fig. 5. Self-oscillatory solutions of the Rayleigh equation for the initial amplitude that is smaller (left) and larger (right) than the limit cycle amplitude. The time is shown in the units of oscillation periods, $2 \pi / C_{\mathrm{K}}$. The units of the vertical axis are arbitrary.

parameter $\Delta$ is positive. This corresponds to the case of the effective negative damping, also known as over-stability. In this case the oscillation amplitude grows exponentially, until it reaches a certain amplitude prescribed by the parameters of the Rayleigh oscillator equation, and corresponding to a limit cycle. Mathematically this effect is controlled by the non-linear term in the square brackets, which accounts for the finite amplitude effects. In the case of a large initial amplitude, the solution decreases to the limit cycle value. For small amplitudes, the limit cycle period coincides with the period $2 \pi / \Omega_{\mathrm{K}}$. Figure 5 demonstrates two scenarios of the excitation of oscillatory solutions of Eq. (3) for the initial amplitude that is larger and smaller than the limit cycle amplitude. After a quick transition, the oscillatory pattern becomes harmonic with the period coinciding with the period $2 \pi / \Omega_{\mathrm{K}}$.

In oscillation theory such a behaviour is known as auto-oscillations, self-sustained oscillations, or simply selfoscillations (Andronov et al. 1996; Jenkins 2013). Selfoscillations occur in non-conservative dissipative systems. In contrast with driven oscillations, a self-oscillator itself sets the frequency and phase with which it is driven, keeping the frequency and phase for a number of periods. Self-oscillations occur in various dynamical systems in physics, engineering, biology, and economics. Usually, parameters of self-oscillations, such as amplitudes, periods, and phases, are independent of the initial excitation of the system after some transition time. Self-oscillations could be considered processes of a production of a periodic signal, for example an alternate current, from a steady energy supply, such as a direct current. A relevant example to our discussion is the response of a violin when the bow is slowly moving across the string. When playing a note, there is some minimum, threshold speed at which the bow must be moved, but moving it faster does not change the note and only makes it louder. The possible analogy between the 
excitation of kink oscillations of coronal loops and a violin was pointed out by Goedbloed (1995).

In the case of kink oscillations the coronal loops play the role of a string, while the role of the bow can be played, for example by supergranulation motions; see Fig. 4 . Indeed, as a characteristic timescale of these motions is several hours and a typical horizontal scale is several tens of Mm (e.g. Rieutord \& Rincon 2010), it can be considered a steady motion around the loop footpoint. Thus, the interaction of the loop with a supergranulation flow could be considered in terms of Eq. (3). The average velocity amplitude of undamped kink oscillations, about $4 \mathrm{~km} \mathrm{~s}^{-1}$ (see Fig. 2, right panels), is detected at the loop tops, where the fundamental kink modes have antinodes, while the interaction with the flow occurs near the footpoints. As the fundamental modes have nodes near the loop footpoints, their velocity amplitudes are very small near the loop footpoints. Thus, it is possible that the threshold value for the onset of negative friction is reached and a self-oscillation occurs. Another possibility would be an upflow passing the coronal part of the loop, which was discussed, for instance in Nakariakov et al. (2009). In this case, the vortex shedding effect should be suppressed, which can take place, among other reasons, because of the external magnetic field.

\section{Discussion and conclusions}

In this Letter we propose a model that interprets the undamped quasi-harmonic kink oscillations of coronal loops as self-oscillations occurring because of the slippery sliding interaction of the loop with a quasi-steady flow across it. The model is based on the Rayleigh ordinary differential equation that has a limit cycle solution corresponding to the self-oscillations. This model successfully reproduces observed properties of this class of kink oscillations. It explains the quasi-monochromaticity of the oscillations, as the oscillation period is determined by the natural kink mode. It is also consistent with the observed linear dependence of the oscillation period on the loop length. Also, this model explains the apparent independence of the oscillation amplitude on the period, as different loops have different periods, and the energy is taken from the steady flow at this specific period and with the specific rate required to compensate the dissipative losses. This is a feature of self-oscillations. Thus, the model also explains the undamped nature of the oscillations in the presence of dissipative and mode conversion processes.

We must stress that the proposed self-oscillatory mechanism is based on a semi-empirical and low-dimensional description, and thus can only be considered as a conceptual model. The governing equation (Eq. (3)) was not rigorously derived from the set of MHD equations, but was constructed accounting for the physical effects that are important for reproducing the observed behaviour. Such low-dimensional, conceptual models appear to be very useful in various applications, for example the self-organised criticality model (e.g. Aschwanden et al. 2016). The proposed model obviously misses a number of other potentially important effects, in particular, the possibility of the excitation of multiple modes and harmonics. These effects need to be included in more advanced models. Also, the nature of the friction force between the external flow and the loop, and the determination of the threshold speed require attention. However, we believe that the proposed conceptual, low-dimensional model provides new insights, and is useful for identifying the basic physical mechanisms for the existence of undamped kink oscillations of coronal loops. The ability of the model to explain observational properties of undamped kink oscillations suggests the need for its further development.
Another interesting potential outcome of this study is its possible relevance to the coronal heating problem. Self-oscillatory motions described by Eq. (3) are essentially dissipative, with the dissipative losses compensated by the continuous energy supply. The dissipated energy is, possibly via mode coupling, converted into heat in the corona. The estimation of this energy may shed light on the coronal energy balance. This estimation needs to account for 2D effects, in particular, in the mode localisation in the external medium (e.g. Goossens et al. 2013) and, hence, cannot be performed in terms of the discussed low-dimensional model.

Other examples of self-oscillatory processes in the solar atmosphere could be a cyclic pattern of chromospheric evaporation, condensation and reheating (e.g. Müller et al. 2003), and non-linear thermal overstability (e.g. Kumar et al. 2016). Also, a class of quasi-periodic pulsations in flaring energy releases that are caused by spontaneous repetitive magnetic reconnection ("magnetic dripping", see e.g. Nakariakov et al. 2010), could be considered in terms of self-oscillations. Investigation of these processes is an interesting future task.

Acknowledgements. This work was supported by STFC consolidated grant ST/L000733/1 (G.N., V.M.N.), the European Research Council under the SeismoSun Research Project No. 321141 (S.A.A., V.M.N.), and the BK21 plus program through the National Research Foundation funded by the Ministry of Education of Korea (D.H.L., V.M.N.).

\section{References}

Andronov, A. A., Vitt, A. A., \& Khaikin, S. E. 1996, in Theory of Oscillators (Oxford: Pergamon Press), 848

Anfinogentov, S., Nisticò, G., \& Nakariakov, V. M. 2013, A\&A, 560, A107

Anfinogentov, S. A., Nakariakov, V. M., \& Nisticò, G. 2015, A\&A, 583, A136

Aschwanden, M. J., Fletcher, L., Schrijver, C. J., \& Alexander, D. 1999, ApJ, 520,880

Aschwanden, M. J., Crosby, N. B., Dimitropoulou, M., et al. 2016, Space Sci. Rev., 198, 47

Berghmans, D., \& Tirry, W. J. 1997, A\&A, 325, 318

De Groof, A., \& Goossens, M. 2000, A\&A, 356, 724

De Groof, A., \& Goossens, M. 2002, A\&A, 386, 691

De Groof, A., Tirry, W. J., \& Goossens, M. 1998, A\&A, 335, 329

Edwin, P. M., \& Roberts, B. 1983, Sol. Phys., 88, 179

Goddard, C. R., Nisticò, G., Nakariakov, V. M., \& Zimovets, I. V. 2016, A\&A, 585, A 137

Goedbloed, J. P. 1995, in AIP Conf. Ser., 345, 465

Goossens, M., Andries, J., \& Aschwanden, M. J. 2002, A\&A, 394, L39

Goossens, M., Van Doorsselaere, T., Soler, R., \& Verth, G. 2013, ApJ, 768, 191 Hindman, B. W., \& Jain, R. 2014, ApJ, 784, 103

Hood, A. W., Ruderman, M., Pascoe, D. J., et al. 2013, A\&A, 551, A39

Jenkins, A. 2013, Phys. Rep., 525, 167

Kumar, S., Nakariakov, V. M., \& Moon, Y.-J. 2016, ApJ, 824, 8

Müller, D. A. N., Hansteen, V. H., \& Peter, H. 2003, A\&A, 411, 605

Nakariakov, V. M., \& Ofman, L. 2001, A\&A, 372, L53

Nakariakov, V. M., Ofman, L., Deluca, E. E., Roberts, B., \& Davila, J. M. 1999, Science, 285,862

Nakariakov, V. M., Aschwanden, M. J., \& van Doorsselaere, T. 2009, A\&A, 502, 661

Nakariakov, V. M., Inglis, A. R., Zimovets, I. V., et al. 2010, Plasma Physics and Controlled Fusion, 52, 124009

Nisticò, G., Nakariakov, V. M., \& Verwichte, E. 2013, A\&A, 552, A57

Nisticò, G., Anfinogentov, S., \& Nakariakov, V. M. 2014, A\&A, 570, A84

Ofman, L., \& Davila, J. M. 1995, J. Geophys. Res., 100, 23427

Pascoe, D. J., Goddard, C. R., Nisticò, G., Anfinogentov, S., \& Nakariakov, V. M. 2016, A\&A, 585, L6

Poedts, S., \& Boynton, G. C. 1996, A\&A, 306, 610

Rayleigh, J. W. S. 1945, The Theory of Sound, Vol. 2 (New York: Dover)

Rieutord, M., \& Rincon, F. 2010, Liv. Rev. Sol. Phys., 7

Ruderman, M. S., \& Roberts, B. 2002, ApJ, 577, 475

Schrijver, C. J., \& Brown, D. S. 2000, ApJ, 537, L69

Uralov, A. M. 2003, Astron. Lett., 29, 486

Wang, T., Ofman, L., Davila, J. M., \& Su, Y. 2012, ApJ, 751, L27

Zaitsev, V. V., \& Stepanov, A. V. 1982, Sov. Astron. Lett., 8, 132

Zimovets, I. V., \& Nakariakov, V. M. 2015, A\&A, 577, A4 